\title{
E studio de la vía auditiva central por medio de las respuestas evocadas auditivas del tronco encefálico (A BR), en niños con retraso en el lenguaje
}

\author{
MANUEL GALLARDO, CARLOS VERA \\ Servicio de Otorrinolaringología, Hospital Central de la Fuerza Aérea del Perú.
}

\begin{abstract}
RESUMEN
OBJETIVO: Determinar la integridad funcional de la vía auditiva en el tronco encefálico a través de las respuestas evocadas auditivas del tronco encefálico (A BR) en niños con retardo en el lenguaje sin patología en oído medio y sistema nervioso central y sin factores de riesgo de hipoacusia neonatal. DISEÑ O: Estudio retrospectivo transversal. LU GAR: Servicios de otorrinolaringología del Centro $\mathrm{M}$ édico $\mathrm{N}$ aval y del Hospital Central de la $\mathrm{F}$ uerza $\mathrm{A}$ érea del Perú. MATERIAL Y MÉTODOS: Se analizó el ABR realizado en los últimos diez años a niños con retardo en el lenguaje, que incluyó 48 niños, 28 varones y 20 mujeres, con edades entre 1 y 11 años. Se evaluó los tiempos de latencia y la morfología de las ondas I, II, III, IV y V del A BR. RESULTADOS: Se encontró las latencias dentro de lo normal para cada edad. En $77 \%$ de los pacientes hubo distorsión morfológica y/o hipovoltaje de las ondas, más frecuentemente (58\%) en las ondas I y II, correspondientes al nervio auditivo y núcleos cocleares en el tronco encefálico. CON CLUSIONES: L os resultados sugieren que existe trastorno en la sincronización neural de la vía auditiva en el VIII par y en el tronco encefálico en $77 \%$ de los pacientes evaluados, Io cual afectaría el procesamiento auditivo central.
\end{abstract}

Palabras clave: Trastornos del desarrollo del lenguaje; potenciales evocados auditivos del tronco cerebral; conductos auditivos.

\section{STUDY OF THE CENTRAL AUDITIVE PATHWAY BY ENCEPHALIC TRUNK EVOKED AUDITORY RESPONSES (EAR) IN CHILDREN WITH LANGUAGE RETARD SUM MARY}

OBJECTIVE: To determine the functional integrity of the brainstem auditory pathway by the auditive brainstem response (ABR) in language-retarded children without pathology in both the middle ear and central nervous system and no neonatal hearing loss risk factors. DESIGN: Retrospective transversal study. SETTING: Naval M edical Center and Air Force Central Hospital Otorhinolaryngology Services, Lima. Peru. MATERIAL AND METHODS: A nalysis of children's A BR performed in the last ten years included 48 children, 28 males and 20 females, 1 to 11 year-old. M ain outcome measures were both latency time and morphology of waves I, II, III, IV, and V. RESU LTS: Latencies were normal at any age. M orphological distortion and/ or wave hypovoltage were found in $77 \%$ of the patients, more frequently $(58 \%)$ in waves I and II that correspond to the auditory nerve and brainstem cochlear nuclei. CONCLUSIONS: The results suggest a disturbance in the neural synchronization of the auditory pathway and the brainstem nuclei in $77 \%$ of the patients studied, that would affect the central auditory processing.

Key words: Language development disorders; evoked potentials, auditory, brain stem; auditory pathways.

Correspondencia:

Dr. Manuel Arturo Gallardo Flores

Servicio de Otorrinolaringología,

Hospital Central de la Fuerza Aérea del Perú

Av. Aramburú. Cuadra $2 \mathrm{~s} / \mathrm{n}$. Lima 18. Perú.

E-mail: anales@sanfer.unmsm.edu.pe 


\section{INTRODUCCIÓN}

Uno de los rasgos característicos del ser humano y que nos diferencia de los animales es la capacidad de comunicarnos a través del lenguaje hablado. El lenguaje se adquiere fundamentalmente en los primeros años de vida.

Para que el lenguaje se desarrolle en forma normal es necesario una audición intacta desde el nacimiento, sistema nervioso intacto, control de estructuras físicas y fisiológicas requeridas para un habla intelegible y estímulo ambiental adecuado ${ }^{1}$ ).

Las variables que más afectan el desarrollo del lenguaje incluyen el tipo de hipoacusia (conductiva, neurosensorial y mixta), el grado de hipoacusia (leve, moderada y severa) y la lateralidad (uni o bilateral). También es importante tener en cuenta la edad de inicio y la edad de identificación del problema $\left({ }^{2}\right)$.

La audición juega un papel crítico en el desarrollo del lenguaje y los ef ectos de una hipoacusia neurosensorial permanente sobre el desarrollo del lenguaje pueden ser considerables $\left(^{3}\right)$.

EI sistema auditivo es activo en el útero y las etapas iniciales de la audición ocurren en la vida prenatal $\left({ }^{4}\right)$. L a maduración de las vías auditivas neurales están en relación al proceso de mielinización del sistema nervioso central.

La sensibilidad al sonido se desarrolla de manera compleja y continúa aún pasados los cinco años de edad, en la mayor parte sin relación a la maduración de las estructuras auditivas periféricas y del tronco encefálico. La capacidad de seleccionar y discriminar frecuencias exhibe cambios en relación a la edad y está bien desarrollada en los infantes. El procesamiento auditivo que se realiza a través de vías cruzadas, los cuales reflejan niveles centrales de análisis, está maduro a la edad de cuatro años $\left({ }^{5}\right)$.

El procesamiento auditivo central es el conjunto de habilidades específicas de los cuales el individuo depende para interpretar lo que escu- cha. Estas habilidades son mediadas por los centros auditivos localizados en el tronco encefálico y en el cerebro. Éstos pueden dividirse en las siguientes áreas: atención, discriminación, asociación, integración y organización de salida $\left(^{6}\right)$.

El procesamiento del habla y del lenguaje es influenciado por factores intrínsecos asociados con características anatómicas, fisiológicas y bioquímicas del sistema nervioso per se y por factores extrínsecos asociados con el estímulo y la naturaleza de la situación de aprendizaje ( ${ }^{7}$ ).

L as hipoacusias conductivas producidas por otitis medias efusivas crónicas o agenesia del conducto auditivo externo y las hipoacusias sensoriales por cocleopatías pueden ocasionar efectos negativos sobre el desarrollo del procesamiento auditivo en niños $\left(^{5}\right)$.

El normal desarrollo y mielinización de la vía auditiva pueden verse afectadas por ciertas condiciones patológicas que son considerados factores de riesgo de hipoacusia neonatal, tales como, historia familiar de hipoacusia, malformaciones craneofaciales, infecciones virales (citomegalovirus, rubéola, sífilis, etc.), prematuridad, hiperbilirrubinemia, anoxia perinatal o sufrimiento fetal y meningitis neonatal $\left({ }^{8}\right)$.

Es importante hacer un diagnóstico precoz de hipoacusia en niños pequeños, porque de esta manera existen mejores posibilidades de rehabilitación de la audición y del lenguaje $\left({ }^{9}\right)$.

En nuestro medio no tenemos estadísticas sobre hipoacusia en recién nacidos. En Estados Unidos se calcula que cada año nacen 4000 niños con hipoacusia profunda y otros $37000 \mathrm{ni}-$ ños nacen con grados menores de hipoacusia, lo cual hace que 6 de cada 1000 neonatos tengan hipoacusia significativa $\left({ }^{10}\right)$.

Actualmente se utiliza las emisiones otoacústicas y las respuestas evocadas auditivas del tronco encefálico (ABR) como parte del estudio de niños con retraso en la adquisición del lenguaje. 
Desde su primera descripción en los años setenta, el A BR ha demostrado su utilidad como parte integral del estudio otoneurológico, con aplicación en otorrinolaringología, neurología y pediatría, por su exactitud, confiabilidad y reproducibilidad. En esta prueba se estudia la morfología, las latencias e interval os de latencias de las ondas I a V. La onda I corresponde a la porción coclear del nervio auditivo, la onda II al núcleo coclear, Ia onda III al complejo olivar superior, la onda IV al Iemnisco lateral y la onda $\mathrm{V}$-que es la más prominente y el principal parámetro para determinar el umbral auditivocorresponde al colículo inferior.

EI ABR nos permite detectar los umbrales auditivos, hacer diagnóstico de maduración del sistema nervioso central, topodiagnóstico y diagnóstico de simulación auditiva $\left({ }^{9,11}\right)$.

El objetivo del presente trabajo es determinar la integridad funcional de la vía auditiva en el tronco encefálico a través del $A B R$, en niños con retardo en el lenguaje y que no presentaban patología en oído medio ni en el sistema nervioso central y tampoco factores de riesgo de hipoacusia neonatal.

\section{MATERIAL Y MÉTODOS}

Se hizo una revisión de los exámenes de A BR realizados en los últimos diez años a los niños que acudieron a los Servicios de Otorrinolaringología del Centro $\mathrm{M}$ édico $\mathrm{N}$ aval y del Hospital Central de la Fuerza A érea del Perú, por presentar retraso en el lenguaje y que además no presentaron patología del oído medio (determinado por impedanciometría) ni del sistema nervioso central; además, no presentaron factores de riesgo de hipoacusia neonatal, tales como prematuridad, hipoxia neonatal, hiperbilirrubinemia, infecciones virales maternas, etc.

Se estudió 48 niños con retraso en el lenguaje y que reunieron estos requisitos. De ellos, 28 $(58 \%)$ fueron varones y $20(42 \%)$ mujeres.
Se evaluó los tiempos de latencia de las ondas I, II, III, IV y V de ambas vías auditivas, las cuales fueron comparadas por edad con los parámetros de normalidad, según el equipo usado en su evaluación. En el Centro $M$ édico $\mathrm{N}$ aval se utilizó un equipo Nicolet, cuyos parámetros de normalidad son los siguientes:

\begin{tabular}{lccccc}
\hline & I & II & III & V & V \\
\hline$<$ 1 año & 1,62 & 2,84 & 3,88 & 4,76 & 5,85 \\
1 a 5 años & 1,59 & 2,78 & 3,84 & 4,69 & 5,82 \\
$>$ 5 años & 1,54 & 2,68 & 3,68 & 4,53 & 5,69 \\
DS $= \pm 0,18$ & & & & & \\
\hline
\end{tabular}

En el Hospital Central FAP se utilizó un equipo Racia, siendo los parámetros de normalidad los siguientes:

\begin{tabular}{lccccc}
\hline & I & II & III & IV & V \\
\hline$<1$ año & 1,74 & 2,97 & 3,93 & 4,82 & 6,10 \\
1 a 5 años & 1,70 & 2,89 & 3,88 & 4,79 & 6,00 \\
$>$ 5 años & 1,59 & 2,72 & 3,75 & 4,56 & 5,85 \\
DS $= \pm 0,20$ & & & & & \\
\hline
\end{tabular}

Evaluamos, además, la morfología de las ondas y su voltaje. Cuando las ondas estaban presentes, pero el trazado era irregular, eran catalogadas como distorsionadas. Si su voltaje era menor de 0,2 uv, era considerada como hipovoltaje.

\section{RESULTADOS}

El $81 \%$ de los pacientes tuvo cuatro años o menos de edad, siendo el grupo de 3 y 4 años el de mayor cantidad de pacientes (56\%).

En la Tabla 1 describimos en detalle el A BR de cada uno de los 48 niños evaluados.

En la Tabla 2 se encuentra consignado el promedio de las Iatencias de las ondas I, III y V 
para cada edad. Estas latencias se encontraban dentro de los límites de normalidad, excepto en el grupo de tres años, en el que había una leve prolongación de las ondas I a V de ambas vías auditivas.
De los 48 pacientes evaluados, un 77\% (36 pacientes) presentó alteración en la morfología y en el voltaje de las ondas de una o ambas vías auditivas, siendo normal el trazado en el $23 \%$ restante.

Tabla 1-. Características del ABR de los pacientes estudiados. $(n=48)$

\begin{tabular}{|c|c|c|c|c|c|c|c|}
\hline Caso & Sexo & Edad & Oído & I & III & V & Morfología \\
\hline \multirow[t]{2}{*}{1} & \multirow[t]{2}{*}{ M } & \multirow[t]{2}{*}{$4 a$} & $\mathrm{D}$ & 1,50 & 3,82 & 5,60 & \multirow{2}{*}{ Hipovoltaje ondas I y II bilateral y distorsión morfológica. } \\
\hline & & & I & 1,50 & 3,98 & 5,90 & \\
\hline \multirow[t]{2}{*}{2} & \multirow[t]{2}{*}{$\mathrm{F}$} & \multirow[t]{2}{*}{$11 \mathrm{a}$} & $\mathrm{D}$ & 1,60 & 4,10 & 6,30 & \multirow{2}{*}{$\begin{array}{l}\text { Hipovoltaje y distorsión morfológica de ondas III y V bilateral. } \\
\text { Hipovoltaje de ondas I y II derechas. }\end{array}$} \\
\hline & & & I & 1,50 & 3,90 & 6,20 & \\
\hline \multirow[t]{2}{*}{3} & \multirow[t]{2}{*}{$\mathrm{F}$} & \multirow[t]{2}{*}{$11 \mathrm{a}$} & $\mathrm{D}$ & 1,50 & 3,70 & 7,20 & \multirow[t]{2}{*}{ Hipovoltaje y distorsión morfológica de ondas I a V bilateral. } \\
\hline & & & I & 1,50 & 3,80 & 6,60 & \\
\hline \multirow[t]{2}{*}{4} & \multirow[t]{2}{*}{ M } & \multirow[t]{2}{*}{$4 \mathrm{a}$} & $\mathrm{D}$ & 1,90 & 4,00 & 6,10 & \multirow[t]{2}{*}{ Hipovoltaje y distorsión morfológica de ondas I a V derechas. } \\
\hline & & & I & 1,50 & 3,90 & 5,70 & \\
\hline \multirow[t]{2}{*}{5} & \multirow[t]{2}{*}{ M } & \multirow[t]{2}{*}{$4 \mathrm{a}$} & $\mathrm{D}$ & 1,62 & 3,54 & 5,92 & \multirow[t]{2}{*}{ Normal. } \\
\hline & & & I & 1,56 & 3,54 & 6,02 & \\
\hline \multirow[t]{2}{*}{6} & \multirow[t]{2}{*}{ M } & \multirow[t]{2}{*}{$5 \mathrm{a}$} & $\mathrm{D}$ & 1,60 & 3,90 & 5,90 & Hipovoltaje y distorsión morfológica de vía auditiva derecha. \\
\hline & & & $\mathrm{I}$ & 1,50 & 3,70 & 5,80 & \\
\hline 7 & M & $5 \mathrm{a}$ & $\mathrm{D}$ & 1,50 & 3,70 & 5,80 & Normal. \\
\hline & & & I & 1,40 & 3,70 & 5,70 & \\
\hline 8 & M & $4 \mathrm{a}$ & $\mathrm{D}$ & 1,80 & 4,00 & 5,90 & Distorsión morfológica de onda I izquierda e hipovoltaje de \\
\hline & & & I & 2,50 & 4,60 & 6,50 & toda la vía auditiva derecha. \\
\hline 9 & $\mathrm{~F}$ & $2 \mathrm{a}$ & $\mathrm{D}$ & 1,40 & 3,50 & 5,40 & Hipovoltaje de la onda I izquierda. \\
\hline & & & I & 1,50 & 3,50 & 5,50 & \\
\hline 10 & M & $3 a$ & $\mathrm{D}$ & - & 4,90 & 6,80 & Ausencia de ondas I y II derechas. \\
\hline & & & I & 2,10 & 4,10 & 6,20 & \\
\hline 11 & $\mathrm{~F}$ & $2 \mathrm{a}$ & $\mathrm{D}$ & 1,50 & 3,80 & 5,70 & Distorsión morfológica de la onda I izquierda e hipovoltaje de \\
\hline & & & I & 1,50 & 3,70 & 5,70 & onda II izquierda. \\
\hline 12 & M & $3 \mathrm{a}$ & $\mathrm{D}$ & 1,60 & 4,20 & 6,10 & Distorsión morfológica de onda II derecha y de ondas I y II \\
\hline & & & I & 2,10 & 3,90 & 6,00 & izquierdas. \\
\hline 13 & $\mathrm{~F}$ & $4 \mathrm{a}$ & $\mathrm{D}$ & 1,66 & 3,62 & 5,68 & Distorsión morfológica e hipovoltaje de toda la vía auditiva \\
\hline & & & I & 1,74 & 3,42 & 5,72 & izquierda. \\
\hline 14 & $\mathrm{~F}$ & $1 \mathrm{a}$ & $\mathrm{D}$ & 1,40 & 3,80 & 5,60 & Hipovoltaje de ondas I y II izquierdas. \\
\hline & & & I & 1,60 & 3,90 & 6,00 & \\
\hline 15 & M & $3 a$ & $\mathrm{D}$ & 1,48 & 3,70 & 6,00 & Hipovoltaje de ondas I a V de la vía auditiva izquierdas. \\
\hline & & & I & 1,90 & 4,02 & 6,18 & \\
\hline 16 & $\mathrm{~F}$ & $6 a$ & $\mathrm{D}$ & 1,50 & 3,60 & 5,50 & Normal. \\
\hline & & & $\mathrm{I}$ & 1,60 & 3,60 & 5,50 & \\
\hline 17 & $\mathrm{~F}$ & $4 a$ & $\mathrm{D}$ & 1,40 & 3,40 & 5,30 & Hipovoltaje de onda I derecha. \\
\hline & & & $\mathrm{I}$ & 1,40 & 3,50 & 5,60 & \\
\hline 18 & M & $6 a$ & $\mathrm{D}$ & 1,60 & 3,64 & 5,56 & Normal. \\
\hline & & & I & 1,58 & 3,56 & 5,60 & \\
\hline 19 & M & $2 \mathrm{a}$ & $\mathrm{D}$ & 1,60 & 3,78 & 5,62 & Distorsión morfológica de ondas I y II izquierdas. \\
\hline & & & I & 1,58 & 3,98 & 5,68 & \\
\hline 20 & $\mathrm{~F}$ & $1 \mathrm{a}$ & $\mathrm{D}$ & 1,66 & 4,08 & 6,36 & Distorsión morfológica de la vía auditiva izquierda y de la \\
\hline & & & I & 1,64 & 4,08 & 6,24 & onda II derecha. \\
\hline 21 & M & $9 \mathrm{a}$ & $\mathrm{D}$ & 1,90 & 4,00 & 6,10 & Hipovoltaje de ondas I a V derechas y ausencia de ondas en \\
\hline & & & I & - & - & - & vía auditiva izquierda. \\
\hline 22 & M & $3 \mathrm{a}$ & $\mathrm{D}$ & 1,60 & 4,00 & 5,80 & Hipovoltaje de todas las ondas de ambas vías auditivas. \\
\hline & & & I & 1,40 & 3,90 & 5,70 & \\
\hline
\end{tabular}




\begin{tabular}{|c|c|c|c|c|c|c|c|}
\hline Caso & Sexo & Edad & Oído & I & III & $\mathrm{V}$ & Morfología \\
\hline 23 & $\mathrm{~F}$ & $4 a$ & $\begin{array}{l}\mathrm{D} \\
\mathrm{I}\end{array}$ & $\overline{1,70}$ & $\begin{array}{l}4,20 \\
3,70\end{array}$ & $\begin{array}{l}6,00 \\
5,80\end{array}$ & $\begin{array}{l}\text { Ausencia de la onda I derecha y distorsión morfológica de la } \\
\text { vía auditiva derecha. }\end{array}$ \\
\hline 24 & M & $4 a$ & $\begin{array}{l}\mathrm{D} \\
\mathrm{I}\end{array}$ & $\begin{array}{l}1,60 \\
1,50\end{array}$ & $\begin{array}{l}3,70 \\
3,60\end{array}$ & $\begin{array}{l}6,10 \\
5,90\end{array}$ & Hipovoltaje de onda II bilateral. \\
\hline 25 & M & $3 a$ & D & $\begin{array}{l}1,90 \\
1,60\end{array}$ & $\begin{array}{l}4,10 \\
3,80\end{array}$ & $\begin{array}{l}6,00 \\
5,70\end{array}$ & Normal. \\
\hline 26 & M & $3 \mathrm{a}$ & $\begin{array}{l}\mathrm{D} \\
\mathrm{I}\end{array}$ & $\begin{array}{l}1,40 \\
1,50\end{array}$ & $\begin{array}{l}3,50 \\
3,50\end{array}$ & $\begin{array}{l}5,20 \\
5,20\end{array}$ & $\begin{array}{l}\text { Distorsión morfológica de la onda V bilateral e hipovoltaje de } \\
\text { la onda II derecha. }\end{array}$ \\
\hline 27 & M & $8 \mathrm{a}$ & D & $\begin{array}{l}1,40 \\
1,40\end{array}$ & $\begin{array}{l}3,90 \\
4,00\end{array}$ & $\begin{array}{l}5,60 \\
5,80\end{array}$ & Distorsión morfológica de ondas I y II derechas. \\
\hline 28 & M & $5 \mathrm{a}$ & $\begin{array}{l}\text { D } \\
\text { I }\end{array}$ & $\begin{array}{c}- \\
1,80\end{array}$ & $\begin{array}{c}- \\
3,90\end{array}$ & $\begin{array}{l}5,60 \\
5,80\end{array}$ & Distorsión morfológica de ondas I a V derechas. \\
\hline 29 & M & $3 a$ & $\begin{array}{l}\mathrm{D} \\
\mathrm{I}\end{array}$ & $\begin{array}{l}1,70 \\
1,60\end{array}$ & $\begin{array}{l}4,00 \\
4,00\end{array}$ & $\begin{array}{l}5,90 \\
5,90\end{array}$ & Normal. \\
\hline 30 & M & $3 a$ & $\begin{array}{l}\text { D } \\
\text { I }\end{array}$ & - & $\begin{array}{c}4,50 \\
-\end{array}$ & $\begin{array}{l}6,20 \\
6,40\end{array}$ & Distorsión morfológica bilateral. \\
\hline 31 & $\mathrm{~F}$ & $4 a$ & $\begin{array}{l}\mathrm{D} \\
\mathrm{I}\end{array}$ & $\begin{array}{l}1,40 \\
1,50\end{array}$ & $\begin{array}{l}3,40 \\
3,50\end{array}$ & $\begin{array}{l}5,40 \\
5,20\end{array}$ & Distorsión morfológica de ondas I a V derechas. \\
\hline 32 & $\mathrm{~F}$ & $4 a$ & $\begin{array}{l}\text { D } \\
\text { I }\end{array}$ & $\begin{array}{l}1,50 \\
1,50\end{array}$ & $\begin{array}{l}3,70 \\
3,60\end{array}$ & $\begin{array}{l}5,40 \\
5,40\end{array}$ & Hipovoltaje de onda II bilateral. \\
\hline 33 & M & $1 \mathrm{a}$ & $\begin{array}{l}\mathrm{D} \\
\mathrm{I}\end{array}$ & $\begin{array}{l}1,50 \\
1,50\end{array}$ & $\begin{array}{l}3,70 \\
3,60\end{array}$ & $\begin{array}{l}5,40 \\
5,40\end{array}$ & Hipovoltaje de onda II bilateral. \\
\hline 34 & $\mathrm{~F}$ & $4 a$ & $\begin{array}{l}\text { D } \\
\text { I }\end{array}$ & $\begin{array}{l}1,60 \\
1,60\end{array}$ & $\begin{array}{l}3,80 \\
3,80\end{array}$ & $\begin{array}{l}5,80 \\
5,80\end{array}$ & $\begin{array}{l}\text { Distorsión morfológica de onda I izquierda e hipovoltaje de } \\
\text { II bilateral. }\end{array}$ \\
\hline 35 & $\mathrm{~F}$ & $3 a$ & $\begin{array}{l}\mathrm{D} \\
\mathrm{I}\end{array}$ & $\begin{array}{l}1,52 \\
1,64\end{array}$ & $\begin{array}{l}3,80 \\
3,86\end{array}$ & $\begin{array}{l}6,24 \\
5,78\end{array}$ & $\begin{array}{l}\text { Distorsión morfológica de todas las ondas e hipovoltaje de la } \\
\text { onda II bilateral. }\end{array}$ \\
\hline 36 & $\mathrm{~F}$ & $3 \mathrm{a}$ & $\begin{array}{l}\mathrm{D} \\
\mathrm{I}\end{array}$ & $\begin{array}{l}1,60 \\
1,48\end{array}$ & $\begin{array}{l}3,82 \\
3,70\end{array}$ & $\begin{array}{l}5,76 \\
5,86\end{array}$ & Distorsión e hipovoltaje de onda II bilateral. \\
\hline 37 & $\mathrm{~F}$ & $2 \mathrm{a}$ & $\begin{array}{l}\mathrm{D} \\
\mathrm{I}\end{array}$ & $\begin{array}{l}1,50 \\
1,50\end{array}$ & $\begin{array}{l}3,70 \\
3,60\end{array}$ & $\begin{array}{l}5,70 \\
5,60\end{array}$ & Hipovoltaje de onda II bilateral. \\
\hline 38 & M & $3 a$ & D & $\begin{array}{l}2,30 \\
2,30\end{array}$ & $\begin{array}{l}4,70 \\
4,80\end{array}$ & $\begin{array}{l}6,90 \\
7,10\end{array}$ & Distorsión de ondas I y II bilateral. \\
\hline 39 & M & $11 \mathrm{a}$ & D & $\begin{array}{l}1,60 \\
1,60\end{array}$ & $\begin{array}{l}3,72 \\
3,72\end{array}$ & $\begin{array}{l}5,52 \\
5,56\end{array}$ & Hipovoltaje de onda II bilateral. \\
\hline 40 & M & $2 \mathrm{a}$ & $\begin{array}{l}\text { D } \\
\text { I }\end{array}$ & $\begin{array}{l}1,44 \\
1,44\end{array}$ & $\begin{array}{l}3,80 \\
3,88\end{array}$ & $\begin{array}{l}5,24 \\
5,84\end{array}$ & Normal. \\
\hline 41 & $\mathrm{~F}$ & $2 \mathrm{a}$ & $\begin{array}{l}\text { D } \\
\text { I }\end{array}$ & $\begin{array}{l}1,68 \\
1,64\end{array}$ & $\begin{array}{l}3,64 \\
3,52\end{array}$ & $\begin{array}{l}5,40 \\
5,04\end{array}$ & Normal. \\
\hline 42 & $\mathrm{~F}$ & $3 a$ & $\begin{array}{l}\mathrm{D} \\
\mathrm{I}\end{array}$ & $\begin{array}{l}1,64 \\
1,44\end{array}$ & $\begin{array}{l}3,80 \\
3,76\end{array}$ & $\begin{array}{l}5,56 \\
5,24\end{array}$ & Distorsión de onda I derecha. \\
\hline 43 & M & $4 a$ & $\begin{array}{l}\text { D } \\
\text { I }\end{array}$ & $\begin{array}{l}1,52 \\
1,52\end{array}$ & $\begin{array}{l}3,72 \\
3,84\end{array}$ & $\begin{array}{l}5,48 \\
5,84\end{array}$ & Normal. \\
\hline 44 & $\mathrm{~F}$ & $4 a$ & $\begin{array}{l}\text { D } \\
\text { I }\end{array}$ & $\begin{array}{l}1,64 \\
1,52\end{array}$ & $\begin{array}{l}3,64 \\
3,56\end{array}$ & $\begin{array}{l}5,28 \\
5,18\end{array}$ & Normal. \\
\hline 45 & M & $4 a$ & $\begin{array}{l}\mathrm{D} \\
\mathrm{I}\end{array}$ & $\begin{array}{l}1,52 \\
1,80\end{array}$ & $\begin{array}{l}3,68 \\
3,80\end{array}$ & $\begin{array}{l}5,48 \\
5,80\end{array}$ & Distorsión de las ondas I a III izquierdas. \\
\hline 46 & M & $2 \mathrm{a}$ & $\begin{array}{l}\text { D } \\
\text { I }\end{array}$ & $\begin{array}{l}1,72 \\
1,48\end{array}$ & $\begin{array}{l}4,12 \\
3,80\end{array}$ & $\begin{array}{l}6,04 \\
5,80\end{array}$ & Normal. \\
\hline 47 & $\mathrm{~F}$ & $4 a$ & D & $\begin{array}{l}1,88 \\
1,64\end{array}$ & $\begin{array}{l}4,28 \\
4,00\end{array}$ & $\begin{array}{l}6,20 \\
6,04\end{array}$ & Distorsión bilateral de la onda II. \\
\hline 48 & M & $2 \mathrm{a}$ & $\begin{array}{l}\text { D } \\
\text { I }\end{array}$ & $\begin{array}{l}1,56 \\
1,56\end{array}$ & $\begin{array}{l}3,76 \\
3,76\end{array}$ & $\begin{array}{l}5,72 \\
5,76\end{array}$ & Distorsión de la onda V izquierda. \\
\hline 49 & M & $2 \mathrm{a}$ & $\begin{array}{l}\text { D } \\
\text { I }\end{array}$ & $\begin{array}{l}1,48 \\
1,44\end{array}$ & $\begin{array}{l}3,96 \\
3,80\end{array}$ & $\begin{array}{l}5,68 \\
5,56\end{array}$ & Normal. \\
\hline
\end{tabular}


Tabla 2.- Promedio de latencias por edad.

\begin{tabular}{ccccc}
\hline Edad & Oído & I & III & V \\
\hline \multirow{2}{*}{$1 \mathrm{a}$} & $\mathrm{D}$ & 1,52 & 3,86 & 5,79 \\
& $\mathrm{I}$ & 1,58 & 3,86 & 5,88 \\
$2 \mathrm{a}$ & $\mathrm{D}$ & 1,54 & 3,78 & 5,59 \\
& $\mathrm{I}$ & 1,52 & 3,74 & 5,61 \\
$3 \mathrm{a}$ & $\mathrm{D}$ & 1,61 & 4,11 & 6,00 \\
& $\mathrm{I}$ & 1,74 & 3,88 & 5,91 \\
$4 \mathrm{a}$ & $\mathrm{D}$ & 1,58 & 3,74 & 5,68 \\
& $\mathrm{I}$ & 1,62 & 3,74 & 5,76 \\
$5 \mathrm{a}$ & $\mathrm{D}$ & 1,55 & 3,80 & 5,77 \\
& $\mathrm{I}$ & 1,57 & 3,77 & 5,77 \\
$6 \mathrm{a}$ & $\mathrm{D}$ & 1,55 & 3,62 & 5,53 \\
& $\mathrm{I}$ & 1,59 & 3,58 & 5,50 \\
$8 \mathrm{a}$ & $\mathrm{D}$ & 1,40 & 3,90 & 5,60 \\
& $\mathrm{I}$ & 1,40 & 4,00 & 5,80 \\
$11 \mathrm{a}$ & $\mathrm{D}$ & 1,57 & 3,84 & 6,34 \\
& I & 1,53 & 3,81 & 6,12 \\
\hline
\end{tabular}

De los 36 pacientes, 58\% estaba en las ondas I y II de ambas vías auditivas, siendo las ondas II (núcleos cocleares) las más afectadas. EI $42 \%$ de las alteraciones restantes se encontró en las ondas III a V (Tabla 3).

Tabla 3. Alteración en la morfología y voltaje del ABR. $(n=36)$

\begin{tabular}{lrrrrr}
\hline \multicolumn{1}{c}{ Onda } & I & II & III & IV & V \\
\hline Lado derecho & 17 & 26 & 11 & 10 & 12 \\
Lado izquierdo & 17 & 22 & 9 & 7 & 10 \\
Total & 34 & 48 & 20 & 17 & 22 \\
$\%$ & 24 & 34 & 14 & 12 & 16 \\
\hline
\end{tabular}

\section{DISCUSIÓN}

La mayor adquisición del lenguaje ocurre durante los primeros cinco años de vida. Para esto es importante que la audición esté intacta desde el nacimiento, que no exista patología en el sistema nervioso central, que las estructuras requeridas para el habla estén controladas y que exista estímulo ambiental adecuado.

El desarrollo normal de la vía auditiva central se ve afectada por hipoxia neonatal, hiperbilirrubinemia, prematuridad, infecciones virales (citomegalovirus, rubéola, sífilis, herpes o toxoplasmosis), aplicación de ototóxicos durante la gestación. Sin embargo, hasta en $30 \%$ no se encuentra una etiología que la justifique $(7,8)$.

Cuando la hipoxia es severa, se asocia además de deficiente desarrollo neuronal, manifestado usualmente por retardo mental y desórdenes neuromusculares ( ${ }^{12}$ ). En el kernícterus, las células ciliadas están normales y la vía auditiva está dañada a nivel de los núcleos cocleares; en algunos pacientes, el nervio auditivo también parece estar dañado $\left({ }^{13}\right)$.

En la mayor parte de los niños con trastornos auditivos centrales, éstos no son consecuencia de condiciones neuropatológicas documentadas. En ellos el deterioro auditivo está relacionado a una disfunción idiopática de la vía auditiva central, la cual es comúnmente referida como trastorno del procesamiento auditivo central $\left({ }^{14}\right)$.

Hemos realizado la revisión del A B R de 48 niños con retraso en la adquisición del lenguaje, los cuales no presentaron patología en el oído medio ni en el sistema nervioso central y que, además, no presentaron factores de riesgo de hipoacusia neonatal. En $77 \%$ de los evaluados se encontró al teración en la morfología y/o en el voltaje de las ondas, pero con tiempos de latencia en general dentro de los parámetros de la normalidad. Stach y colaboradores, en un estudio realizado en 44 niños en quienes se diagnosticó trastorno del procesamiento auditivo central, reportaron que el $A B R$ fue normal en todos los niños, pero en $84 \%$ hubo anormalidad en los potenciales evocados de latencia tardía ${ }^{\left({ }^{15}\right)}$. E sto debido a que 
ellos evaluaron los tiempos de latencia y no la morfología de las ondas.

Cuando se realiza el estudio del ABR, debe evaluarse tiempo de latencias, morfología y voltaje de las ondas. E stas últimas variables del A BR (morfología y voltaje) son dependientes de la sincronización neural del VIII par craneal en el tronco encefálico $\left({ }^{16}\right)$.

El hecho de la presencia de distorsión en la morfología de las ondas nos indica que, en nuestros pacientes existe una desincronización neural en la vía auditiva central, más frecuentemente a nivel de la porción distal del nervio auditivo y de los núcleos cocleares en el tronco encefálico.

L a importancia de la identificación temprana de esta patología radica en que se puede instaurar en forma precoz la rehabilitación del lenguaje, con resultados terapéuticos más favorables.

En resumen, en nuestro estudio $58 \%$ de los niños evaluados fue varón y $56 \%$ de la población de estudio tenía entre 3 y 4 años de edad. Los tiempos de latencia estuvieron dentro de lo normal, presentándose distorsión morfológica e hipovoltaje de las ondas I a $\mathrm{V}$ en $77 \%$ de los pacientes. En 58\% de las alteraciones de la morfología y voltaje, ésta se encontró en las ondas I y II, correspondientes a la porción coclear del nervio auditivo y los núcleos cocleares respectivamente. En los niños con retraso en el lenguaje y que carecían de patología en oído medio y en el sistema nervioso central, y que además carecían de factores de riesgo de hipoacusia neonatal, la afección se encontró en la morfología y el voltaje de las ondas y no en los tiempos de latencia, lo cual indica un posible trastorno en la sincronización neural de la vía auditiva.

\section{B IBLIOGRAFÍA}

1. Nelson WE. Tratado de Pediatría. 9a Edición. M éxico: Editorial Interamericana. 1986.

2. Roberts J, Wallace I. D evel opment of speech and language. En: Lalwani AK, Grundfast KM. Pediatric, Otology and Neurotology. Philadelphia: L ippincott-R aven. 1998.

3. Merer DM, Gravel J S. Screening infants and young children for hearing loss: Examination of the CAST procedure. J A m A cademy A udiology 1997; 4: 233-42.

4. Hepper PG, Shadidullah BS. D evel opment of fetal hearing. Arch Dis Child 1994; 71: 81-7.

5. Grose JH, Hall III J W. A uditory development. En: L alwani AK, Grundfast KM. Pediatric, Otology and Neurotology. Philadel phia: L ippincott-R aven. 1998.

6. A costa Alvarez AM, Balen SA, M isorelli MI. Processamento auditivo central: Proposta de avaliacao e diagnostico diferencial. En: Lei Munhos MS, Garcia da Silva ML, Caovilla HH. Audiologia Clinica. Sao Paulo: A theneu. 2000.

7. Bever J. Language and perception. En: Miller G. Communication, language and meaning. N ew Y ork: Basic Books Inc. 1973.

8. Joint Committee on Infant Hearing Position Statement. Pediatrics 1982; 70: 496.

9. Bogacz J . L os potenciales evocados en el hombre. Significación y aplicaciones clínicas. Buenos Aires: El A teneo. 1985.

10. Northern J L, E pstein S. N eonatal hearing screening early identification. En: Lalwani AK, Grundfast KM. Peditric Otology and N eurotology. Philadelphia: Lippincott-Raven. 1998.

11. Lehnhardt E. Práctica de la audiometría. Buenos A ires: Editorial M édica Panamericana. 1992.

12. Bergman I, Hish R P, Fria TJ . Cause of hearing loss in the high risk premature infant. J Pediatr 1985; 106: 95-101.

13. Ahdab-Barmada M, Moossy J. The neuropathology of kernicterus in the premature neonate: diagnostic problems. J Neuropathol Exp Neurol 1984; 43: 45-56.

14. Stach BA. Central Auditory Disorders. En: Lalwani AK, Grundfast KM. Pediatric, Otology and Neurotology. Philadel phia-N ew Y ork: Lippincott-Raven, 1998.

15. Stach BA, Loiselle L H, Jerger J F. A uditory evoked potential abnormalities in children with central auditory disorders. ASHA 1988; 30: 133 (A bstract).

16. Tapia M C. N europatía A uditiva Infantil. Rev electrónica A udiología V ol 1, 2001. http://www.auditio.com 